\title{
Encrypted Color Image Transmission in an Amplify and Forward Relaying Based STBC Encoded Cooperative MIMO OFDM System
}

\author{
K. J. A. CHISTY ${ }^{1}$, S. M. A. ISLAM ${ }^{2}$, S. E. ULLAH ${ }^{3}$, S. R. SABUJ ${ }^{4}$ \\ ${ }^{1,2}$ Department of Physics, Jahangirnagar University \\ ${ }^{3}$ Department of APEE, University of Rajshahi \\ ${ }^{4}$ Department of EEE, Bangladesh University \\ E-mail: ${ }^{1}$ kja_chisty@yahoo.com, ${ }^{2}$ azharphyd@yahoo.com, ${ }^{3}$ enayet67@yahoo.com, ${ }^{4}$ saifuriict@gmail.com

\begin{abstract}
The present paper emphasizes greatly on the development of a simulation model for the single relaying based cooperative MIMO OFDM wireless communication system This paper provides a new insight on system performance comparison with Amplify and Forward relaying protocol, STBC and Spatial multiplexing schemes, four channel equalization techniques (ZF, MMSE, ZF-SIC and MMSE-SIC) and four digital modulation schemes (BPSK, QPSK, 16PSK and 64PSK). From simulation results for encrypted color image transmission, it is observable that the STBC encoded MIMO-OFDM system with Amplify and Forward relaying protocol, BPSK digital modulation and MMSE-SIC channel equalization technique provides an encouraging and quite acceptable system performance.
\end{abstract}

Keywords: Cooperative communication, Orthogonal frequency division multiplexing, Amplify and forward, Minimum mean square error and Zero forcing.

\section{INTRODUCTION}

The increasing demands for providing wireless multimedia and interactive high speed internet services are fuelling intensive research efforts to materialize the vision of future/next generation Internet Protocol (IP)-based heterogeneous multiantenna supported robust networks (Relaying and Cooperative transmission technology) based LTEadvanced/ Multiple antenna supported OFDM and its siblings. Now a days, the people are using their personal wireless enabled devices such as cell phones, laptops and PDAs to communicate with others residing in different parts of the world with existing $2 \mathrm{G}, 3 \mathrm{G}$ and $\mathrm{B} 3 \mathrm{G}$ (up to $3.9 \mathrm{G}$ ) networks based on implemented WCDMA/HSPA, GSM/EDGE, TD-SCDMA and CDMA2000, WiMAX (Fixed and Mobile) and 4G LTE radio access technologies $[1,3]$.

Recently, cooperative communication has attracted to considerable attentions for their great achievements in wireless networks. Various types of cooperative communication have been proposed to achieve the goal such as amplify and forward (AF), decode and forward (DF), fixed relaying (FR), selection relaying (SR), coded cooperation (CC) and compress and forward (CF) were introduced in $[4,7]$ respectively. The benefit of $\mathrm{AF}$ relay protocol is its simplicity and low cost implementation. DF relays which decode and possibly re-encode the information before transmission it and AF relays which forward the signal without hard decoding [8]. M. M. Hossain et al. made a comprehensive study elucidating the performance of a single relay cooperative OFDM system under $\mathrm{AF}$ and $\mathrm{DF}$ relaying strategies on Color image transmission. The results of BER simulation in AWGN and Raleigh fading channels show that the AF relaying protocol supported cooperative OFDM system outperforms in ERC signal combining scheme as compared to others (FRC, SNRC and ESNRC) under BPSK digital modulation [9]. J. J. Sadique et al. presented for a convolutionally encoded MIMO SC-FDMA 
wireless communication system on encrypted color image transmission and the implementation of QAM digital modulation technique with ZF channel equalization provides satisfactory result for convolutionally encoded MIMO SC- FDMA wireless communication system [10]. A. Haroon et al. examined the region of interest (ROI)-based scheme for image transmission over the cooperative wireless channels which improves the performance of ROI compared to direct communication (noncooperative) and conventional cooperative communications image transmission schemes [11].
In this paper, we have established a simulation model for cooperative MIMO-OFDM with single relay implementation of $\mathrm{AF}$ scheme. The revolutionary concept of space time block coding (STBC) introduced in the last decade that the deployment of multiple antennas at the transceiver has been included cooperative MIMO-OFDM scheme. The performance of the system for the zero-forcing (ZF) and minimum mean square error (MMSE), successive interference cancellation (SIC) based minimum mean square error (MMSESIC) and zero forcing (ZF-SIC) has been evaluated in terms of BER and color image.

\section{SYSTEM MODEL}

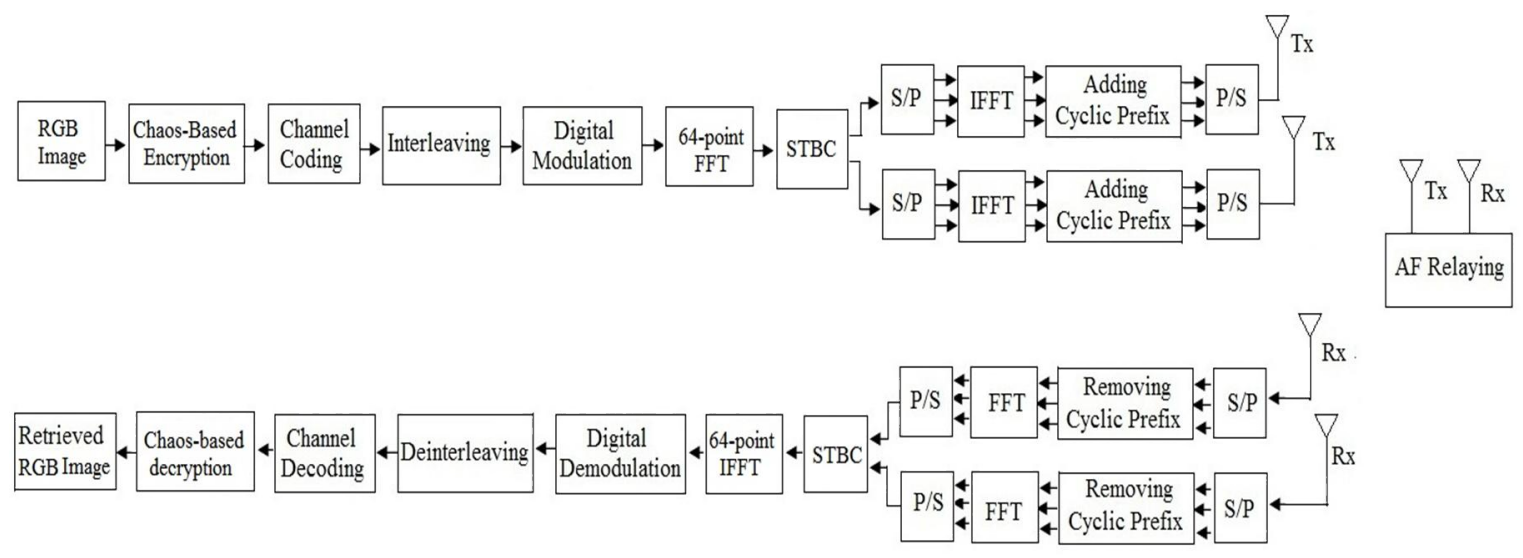

Fig. 1: Block diagram of Single Amplify and Forward Relayed STBC encoded Cooperative MIMO-OFDM system

A cooperative MIMO-OFDM wireless communication system with single relay is shown in Fig. 1. It consists of two users, one user acts as the source while the other user serves as the relay and the two users interchange their message/information as source and relay at different instants in time. A RGB color image in JPEG format is encrypted using chaos based image encryption technique and thereby protecting data error in the simulated system. To meet up such requirement, a simple convolution encoder of rate $1 / 2$ is used in channel coding scheme. The interleaved binary data are converted into digitally modulated signal using BPSK, QPSK, 16PSK and 64PSK. The digitally modulated symbols are then applied to 64-point FFT block and data are rearranged according to the space time block code. On the first antenna the original data and negative second conjugate data is transmitted, while the second antenna the conjugate original data and second data is transmitted [12, 13]. For each transmitting antenna section, the data are passed through serial to parallel converter $(\mathrm{S} / \mathrm{P})$ and transformed into time domain signal using inverse fast Fourier transform (IFFT). A cyclic prefix is then added and is a copy of the last elements of the frame. After parallel to serial converter $(\mathrm{P} / \mathrm{S})$, information symbols are simultaneously transmitted from each transmitting antenna. In receiving end, signals are received in two phases (phase I-direct link and phase II-relay link). In phase I, the data are transferred from source to destination and source to relay directly. On the other hand, in phase II data are transferred from relay to destination. Each cooperative relay has to amplify its received signal and forward its amplified signal through a wireless channel to the destination. In the destination section, the received signals are subsequently passed through OFDM demodulator, 64-point IFFT, digital demodulation, deinteleaver, channel decoder and decryption. Finally the transmitted color image is retrieved.

\section{THEORETICAL ANALYSIS OF SINGLE RELAY}

We assume a RGB color image $(P)$ containing $M$ $x N$ array of color pixels for each of the three $M x N$ sized color image components (Red $\left(P_{r}\right)$, Green $\left(P_{g}\right)$ and Blue $\left(P_{b}\right)$. The range of the color pixel 
value is $[0,255]$. Another secret key image $(K)$ with $\mathrm{M} \mathrm{x} \mathrm{N}$ for the same sized matrix of color image and its matrix components are $K_{r}, K_{g}$ and $K_{b}$ respectively and the same range of pixel value. Each pixel/element of color image compone$\mathrm{nt} /$ matrix component is represented by 8 bits binary digit [0/1]. Then, a diffusion operation is performed to produce cipher image $C$ with components as [14]

$C_{r}=P_{r} \oplus K_{r}$

$C_{g}=P_{g} \oplus K_{g}$

$C_{b}=P_{b} \oplus K_{b}$

Where $\oplus$ is the XOR operation between each pixel of color image and secret key image. Then, we get a binary data, $d=\left[C_{r}, C_{g}, C_{b}\right]$. Binary data stream are fed into a convolutional encoder. After convolutional encoding with redundant binary bit addition, the length of the channel encoded binary data is double. The channel encoded binary data are digitally modulated and FFT conversion. Then, the data are rearranged using STBC $[12,13]$ For the first antenna, $X_{k}^{T, \mathrm{~d}}=\left[X_{0},\left(-X_{1}\right)^{*}, X_{2},\left(-X_{3}\right)^{*} \ldots \ldots\left(. . . X_{K-1}\right)^{*}\right]$ and the second antenna, $X_{k}^{T \times 2}=\left[X_{1},\left(X_{0}\right)^{*}, X_{3},\left(X_{2}\right)^{*} \ldots \ldots . . X_{K-1}\right]$. Then the data sent into serial to parallel converter $(\mathrm{S} / \mathrm{P})$ with 1024 symbols $(\mathrm{K}=1024)$ is processed in each OFDM block to transform into time domain signal using inverse fast Fourier transform (IFFT). The signal is passed through a discrete-time baseband channel with response $h(m)$ and additive white Gaussian noise (AWGN) $w(m)$ is added. In amplify-and-forward (AF) relay scheme, the relay receives a signal from the source; the received signal is amplified and forwarded to the destination in spite of the source-relay link quality. In Phase I, the source-destination and the source-relay received signals are transmitted.

In Phase II, the relay employs a linear precoder F on the received signal vector $r_{s, r}(m)$. The $M_{r} \times M_{r}$ precoding matrix $\mathrm{F}$ is given by $[15,16$, 17]

$F=\sqrt{\frac{1}{\operatorname{tr}\left(H_{s, r}^{H} H_{s, r}\left(\sigma_{r}^{2} I_{M_{r}}+\frac{P_{s}}{M_{s}} H_{s, r}^{H} H_{s, r}\right) H_{r, d} H_{r, d}^{H}\right)}} H_{s, r}^{H} H_{r, d}^{H}$

where, $H_{s, r}$ and $H_{r, d}$ are source to relay and relay to destination channel matrices; $\sigma_{r}^{2}, M_{r}$ and $M_{s}$ are noise variance at relay, no of receiving antenna at relay and no of transmitting antenna at sender respectively. Therefore, the signal transmitted by the relay is given by

$x_{r}(m)=F . r_{s, r}(m)$

By combining the signals received at the destination in both phases, we obtain that $Y(k)$ is the received signal and $H$ is the channel matrix and $N$ is the AWGN for transmitted signal $X_{l}^{T x}$. Under such consideration, received signal $Y(k)$ can be represented as

$Y(k)=H X_{l}^{T x}+N$

\subsection{ZF Signal Detection}

ZF detectors reverse the channel matrix. The Zero Forcing technique nullifies the interference by the following weight matrix $[18,19]$

$$
W_{Z F}=\left(H^{H} H\right)^{-1} H^{H}
$$

Where $(.)^{H_{l}}$ denotes the Hermitian transpose operation. In other words, it inverts the effect of channel as

$x_{Z F}=W_{Z F} Y(k)$

\subsection{MMSE Signal Detection}

The MMSE detector employs a linear filter that can take into account the noise. The MMSE filter is found by minimizing the mean-square error (MSE) as $[18,19]$

$$
W_{\text {MMSE }}=\left(H^{H} H+\sigma_{N_{k}}^{2} I\right)^{-1} H^{H}
$$

With a high SNIR, the MMSE detection becomes the ZF detection. Using the MMSE weight in equation (4), we obtain the following relationship

$$
x_{\text {MMSE }}=W_{M M S E} Y(k)
$$

\subsection{ZF-SIC Detection}

$H$ undergoes $Q R$ factorization as $[18,19]$

$H=Q R=Q\left[\begin{array}{ll}r_{1,1} & r_{1,2} \\ 0 & r_{2,2}\end{array}\right]$

Where, $Q$ and $R$ are the unitary and upper triangular matrix respectively. On multiplying received signal $Y(k)$ of Equation (4) by $Q^{H}$, we can write its product as

$$
\begin{aligned}
x & =Q^{H} Y(k) \\
& =R X_{l}^{T x}+Q^{H} N
\end{aligned}
$$


Whereas, $Q^{H} N$ is a zero-mean complex Gaussian random vector. Since $Q^{H} N$ and $\mathrm{N}$ have the same statistical properties, $Q^{H} N$ can be used to denote N. We get Equation (10) as

$\left[\begin{array}{l}x_{1} \\ x_{2}\end{array}\right]=\left[\begin{array}{ll}r_{1,1} & r_{1,2} \\ 0 & r_{2,2}\end{array}\right]\left[\begin{array}{l}x_{l_{1}} \\ x_{l_{2}}\end{array}\right]+\left[\begin{array}{l}N_{1} \\ N_{2}\end{array}\right]$

The detected desired signal $\hat{X}_{l_{1}}$ from the transmitting antennas can written on neglecting noise term from Equation (11) as

$$
\begin{aligned}
& \hat{X}_{l_{1}}=\frac{\left\{x_{1}-r_{1,2}\left(x_{2} / r_{2,2}\right)\right\}}{r_{1,1}} \text { and } \\
& \hat{X}_{l_{2}}=\frac{x_{2}}{r_{2,2}}
\end{aligned}
$$

\subsection{MMSE-SIC Detection}

The received signal, channel matrix and noise are extended as $[18,19]$

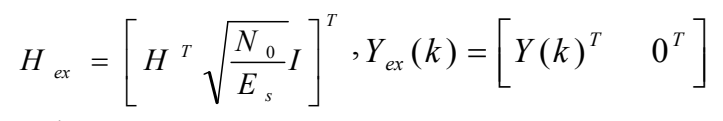

and

$$
N_{e x}=\left[N^{T}-\sqrt{\frac{N_{0}}{E_{s}}} X_{l}^{T}\right]^{T}
$$

Where, $\frac{N_{0}}{E_{s}}=\frac{1}{S N R}$. On QR factorization of extended channel matrix $H_{e x}$, we get

$$
H_{e x}=Q_{e x} \cdot R_{e x}
$$

Where, $Q_{e x}$ and $R_{e x}$ represent a unitary matrix and an upper triangular matrix respectively. We assume that $Y(k), H, N, Q$ and $R$ are replaced by $Y_{e x}(k), H_{e x}$, $N_{e x}, Q_{e x}$ and $R_{e x}$ respectively and correspondingly the resulting system takes the following form

$$
\begin{aligned}
x_{e x} & =Q_{e x}^{H} \cdot Y_{e x}(k) \\
& =R_{e x} \cdot X_{l}^{T x}+Q_{e x}^{H} \cdot N_{e x}
\end{aligned}
$$

Neglecting $Q_{e x}^{H} N_{e x}$ term, the detected desired signal $\hat{X}_{l_{1}}$ from the transmitting antennas can be written from Equation (15) as

$$
\hat{X}_{l_{1}}=\frac{\left\{x_{e x 1}-r_{e x 1,2}\left(x_{e x 2} / r_{e x 2,2}\right)\right\}}{r_{e x 1,1}} \text { and }
$$

$\hat{X}_{l_{2}}=\frac{x_{e x 2}}{r_{e x 2,2}}$

To retrieve the transmitted color image $(\mathrm{P})$ reverse diffusion operation is performed to decrypt the color images with components as,

$P_{r}($ retrieved $)=C_{r} \oplus K_{r}$

$P_{g}($ retrieved $)=C_{g} \oplus K_{g}$

$P_{b}($ retrieved $)=C_{b} \oplus K_{b}$

\section{RESULTS AND DISCUSSION}

This section presents and discusses on the results obtained by the computer simulation program written in MATLAB. Computer simulations have been made to estimate the BERs on color image transmission. The channel coding, FFT/IFFT size and $\mathrm{CP}$ length have been set to be 1/2 -rated Convolutional Encoder, 1024 and 103 symbols respectively.

The BER performance results are presented in Fig. 2 with various digital modulations and MMSE signal detection techniques. The simulated system is found to have shown most satisfactory performance in BPSK digital modulation as compared to other modulations. At a typically assumed SNR value of $6 \mathrm{~dB}$, the BERs of BPSK and QPSK are 0.010 and 0.0245 respectively which implies a system performance improvement of 3.89 dB.

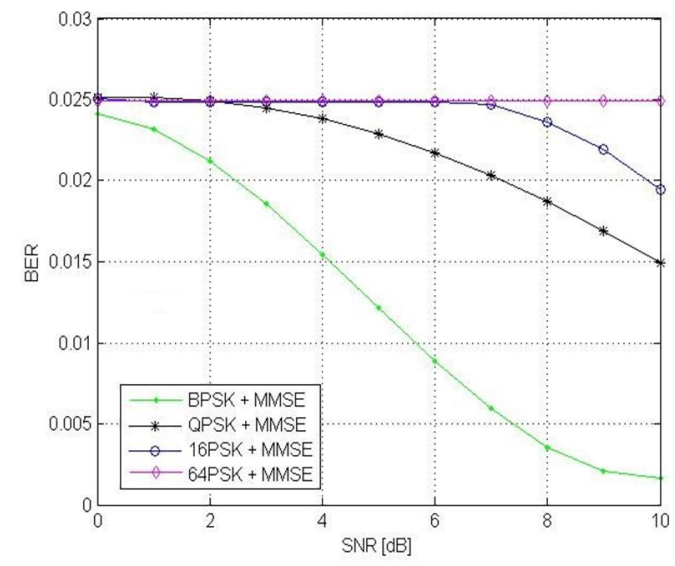

Fig. 2. BER performance of a convolutionally encoded and single relayed $S T B C$ encoded Cooperative MIMO OFDM wireless communication system with MMSE aided signal detection and various digital modulation schemes

In Fig. 3 and 4, we have shown BER performance with implementation of ZF, ZF-SIC, MMSE and MMSE-SIC signal detection schemes for $2 \times 2$ 
K. J. A. Chisty et al. / International Journal of Computer Networks and Communications Security, 1 (7), December 2013

spatially multiplexed cooperative MIMO OFDM and STBC encoded cooperative MIMO OFDM systems. In each case, signaling with BPSK digital modulation, the system shows satisfactory performance. In Fig. 3, it is observed that BER value reduces from 0.026 to 0 when SNR is increased from $0 \mathrm{~dB}$ to $7 \mathrm{~dB}$ in both MMSE and MMSE-SIC signal detection. On the other hand, similar system performance is achieved when SNR is increased from $0 \mathrm{~dB}$ to $10 \mathrm{~dB}$ in $\mathrm{ZF}$ and ZF-SIC signal detection. In Fig. 4, it is seen that the BER value approaches zero at $5 \mathrm{~dB}$ SNR in MMSE-SIC signal detection and in case of ZF-SIC, identical system performance viz. BER approaches zero at 9 dB SNR.

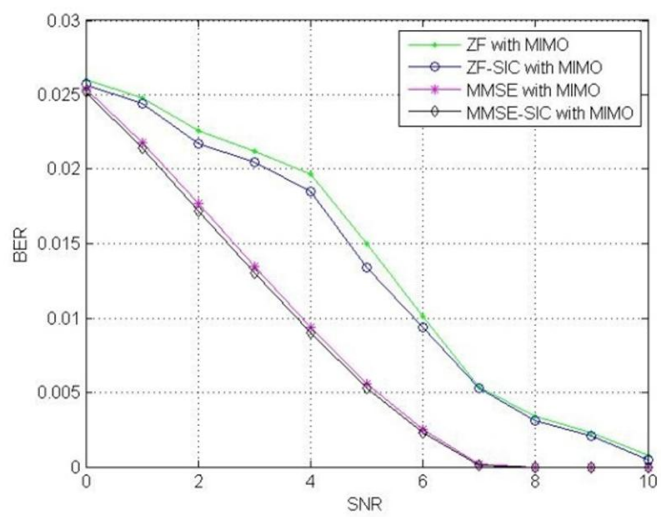

Fig. 3. BER performance comparison of a convolutionally encoded and single relayed Spatially multiplexed Cooperative MIMO OFDM wireless communication system with ZF, MMSE, ZF/MMSE-SIC, aided signal detection and BPSK digital modulation

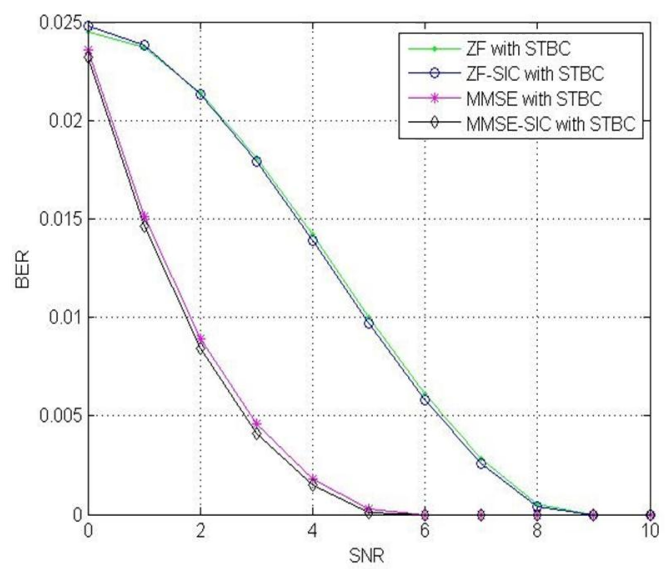

Fig. 4. BER performance comparison of a convolutionally encoded and single relayed STBC encoded Cooperative MIMO OFDM wireless communication system with ZF, MMSE, ZF/MMSE-SIC, aided signal detection and BPSK digital modulation

In Fig. 5, the transmitted and retrieved color images for MMSE and ZF channel equalization, STBC and spatial multiplexing and BPSK digital modulation schemes and numerical results are presented in Table 1.

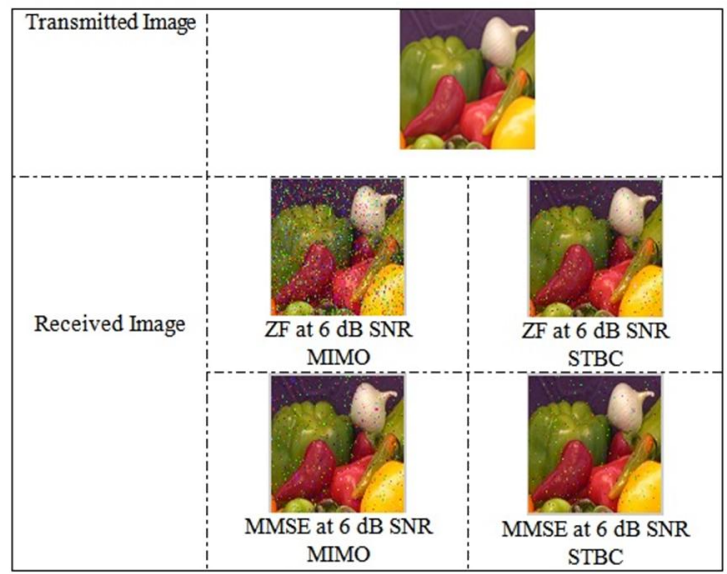

Fig. 5: Transmitted and Received Images for ZF and MMSE channel equalization schemes

Table 1: Comparison of number of error detected and BERs in $Z F$ and MMSE signal detection

\begin{tabular}{|l|c|c|}
\hline \multicolumn{1}{|c|}{ Scheme } & $\begin{array}{c}\text { Number } \\
\text { of Error }\end{array}$ & \multicolumn{1}{c|}{ BER } \\
\hline $\begin{array}{l}\text { ZF with MIMO } \\
\text { (Spatial multiplexing) }\end{array}$ & 201 & $9.0875 \times 10^{-4}$ \\
\hline $\begin{array}{l}\text { MMSE with MIMO } \\
\text { (Spatial multiplexing) }\end{array}$ & 196 & $8.8614 \times 10^{-4}$ \\
\hline ZF with STBC & 73 & $3.3004 \times 10^{-4}$ \\
\hline MMSE with STBC & 39 & $1.7632 \times 10^{-4}$ \\
\hline
\end{tabular}

In Fig. 6, it is observable that the encrypted image is completely not recognizable under implementation of MMSE-SIC and BPSK digital modulation schemes. At a typical case of identical signal and noise power viz. $0 \mathrm{~dB}$, the noisy retrieved image is easily understandable and robustness of the system performance is justified in terms of retrieving signal over hostile environment. In other case of larger signal power as compared to noise power ( $2 \mathrm{~dB}$ and $6 \mathrm{~dB})$, a quite satisfactory system performance is achieved.

\section{CONCLUSION}

In this paper, the bit error rate performance of an AF protocol based cooperative MIMO OFDM wireless communication system has been analyzed and compared under various digital modulations, STBC, Spatial multiplexing, channel coding and channel equalization schemes. In context of system performance, it can be concluded that AF protocol based and STBC encoded cooperative MIMO OFDM wireless communication system provides 
robust and satisfactory performance with MMSESIC signal detection, BPSK digital modulation and 1/2-rated convolutional coding schemes.

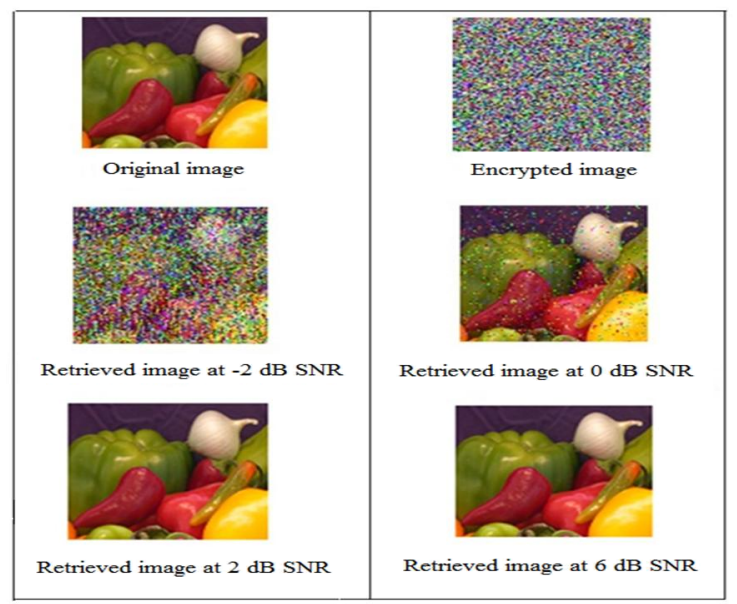

Fig. 6: 2-dimentional graphical illustration showing original, encrypted and retrieved color images at various SNR values

\section{REFERENCES}

[1] G. de la Roche, A. A. Glazunov and B. Allen, "LTE-advanced and next generation wireless networks Channel modelling and propagation" John Wiley and Sons Limited, United Kingdom, 2013.

[2] P. E. Mogensen, T. Koivisto, K. I. Pedersen, I. Z. K. B. Raaf, K. Pajukoski and M. J. Rinne, LTE-Advanced: The Path towards Gigabit/s in Wireless Mobile Communications, IEEE Wireless VITAE, 2009, pp. 147-151.

[3] M. Dottling, W. M. A. Osseiran, Radio Technologies And Concepts For IMTAdvanced, John Wiley and Sons Limited, London, United Kingdom, 2009, pp.1-2.

[4] E. V. D. Meulen, "Three-terminal communication channels," Advances in Applied Probability, vol. 3, 1971, pp. 120-154.

[5] T. M. Cover and A. A. El Gamal. "Capacity theorems for the relay channel" IEEE Trans. Inf. Theory, vol. 25, Sept. 1979, pp. 572-584.

[6] M. Janani, A. Hedayat, T. E. Hunter, and A. Nosratinia, "coded cooperation in wireless communications: space-time transmission and iterative decoding" IEEE Transactions on Signal Processing, vol. 52, February 2004, pp. 362-371.

[7] J. N. Laneman, D. N. C. Tse, and G. W. Wornell, "Cooperative Diversity in Wireless Networks: Efficient Protocols and Outage Behavior" IEEE Trans. Inf. Theory, vol. 50, no. 12 , Dec. 2004, pp. 3062-3080.
[8] Diomidis S. Michalopoulos, A. Suraweera, George K. Karagiannidis, "Amplify-andForward Relay Selection with Outdated Channel Estimates" IEEE transactions on communications, vol.60, no. 5, May 2012.

[9] M M. Hossain and S. E. Ullah, "Performance Evaluation of a Cooperative OFDM System with implementation of DAF and AAF Relaying Protocols on Color image transmission" International Journal of Information Technology Convergence and Services (IJITCS), Vol. 1, No. 3, June 2011, pp. 35-45.

[10]J. J. Sadique and S. E. Ullah, "Performance Analysis of SC-FDMA Wireless Communication System with Implementation of Chaos-Based Image Encryption Scheme" International Journal of Future Generation Communication and Networking, pp. 85-93, Vol. 6, No. 3, June 2013.

[11]A. Haroon, Wireless Imaging Using Cooperative Diversity of Relay Networks, M. Sc Thesis, Blekinge Institute of Technology, April 2010.

[12] S. M. Alamouti, "A simple transmit diversity technique for wireless communications," IEEE Journal on Selected Areas in Communication, Vol. 16, 1998, pp. 1451-1458.

[13] V. Tarokh, H. Jafarkhani, and A. R. Calderbank, "Space-Time Block Coding for Wireless Communications: Performance Results" IEEE Journal on Selected Areas in Communications, vol. 17, no. 3, March 1999, pp. 451-460.

[14]N. A. Al-Romema1, A. S. Mashat and I. AlBidewi, "New Chaos-Based Image Encryption Scheme for RGB Components of Color Image", Computer Science and Engineering, Vol. 2, No. 5, 2012, pp. 77-85.

[15]P. U. Sripathi and J. S. Lehnert, "A throughput scaling law for a class of wireless relay networks" in Proc. 38th Annual Asilomar Conference on Signals, Systems and Computers, Vol. 2, Nov. 2004, pp. 1333-1337.

[16]X. Tang and Y. Hua, "Optimal waveform design for MIMO relaying" In Proceedings of IEEE 6th Workshop on Signal Processing Advances in Wireless Communications, 2005, pp. 289-293.

[17] Y.-W. Peter Hong, Wan-Jen Huang, C.-C. Jay Kuo "Cooperative Communications and Networking, Technologies and System Design" Springer publishing Company, New York, USA, 2010.

[18] Y. S. Cho, J. Kim, W. Y. Yang and C. G. Kang, "MIMO-OFDM Wireless 
K. J. A. Chisty et al. / International Journal of Computer Networks and Communications Security, 1 (7), December 2013

Communications with Matlab" John Wiley and Sons (Asia) Pte Ltd, Singapore, 2010.

[19]L. Bai and J. Choi, "Low Complexity MIMO Detection", Springer Science and Business Media, LLC, New York, USA, 2012. 